\title{
Development and clinical validation of a novel six-gene signature for accurately predicting the recurrence risk of patients with stage II/III colorectal cancer
}

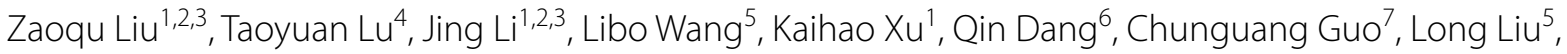
Dechao Jiao', Zhenqiang Sun ${ }^{6^{*}}$ and Xinwei $\operatorname{Han}^{1,2,3^{*}}$ (1)

\begin{abstract}
Background: A large number of patients with stage II/III colorectal cancer (CRC) have a high recurrence rate after radical resection. We aimed to develop a novel tool to stratify patients with different recurrence-risk for optimizing decision-making in post-operative surveillance and therapeutic regimens.

Methods: We retrospectively enrolled four independent cohorts from the Gene Expression Omnibus and 66 CRC tissues from our hospital. The initial signature discovery was conducted in GSE143985 $(n=91)$. This was followed by independent validation of this signature in GSE17536 $(n=111)$, GSE29621 $(n=40)$, and GSE92921 $(n=59)$. Further experimental validation using qRT-PCR assays $(n=66)$ was performed to ensure the robustness and clinical feasible of this signature.

Results: We developed a novel recurrence-related signature consisting of six genes. This signature was validated to be significantly associated with dismal recurrence-free survival in five cohorts GSE143985 (HR: 4.296 [2.612-7.065], $P<0.0001$ ), GSE17536 (HR: 2.354 [1.662-3.334], $P<0.0001$ ), GSE29621 (HR: 3.934 [1.622-9.539], $P=0.0024)$, GSE92921 (HR: 7.080 [2.011-24.924], $P=0.0023$ ), and qPCR assays (HR: 3.654 [2.217-6.020], $P<0.0001$ ). This signature was also proven to be an independent recurrent factor. More importantly, this signature displayed excellent discrimination and calibration in predicting the recurrence-risk at 1-5 years, with most AUCs were above 0.9 , average $C$-index for the five cohorts was 0.8795 , and near-perfect calibration.
\end{abstract}

Conclusions: We discovered and experimental validated a novel gene signature with stable and powerful performance for identifying patients at high recurrence-risk in stage II/III CRC.

Keywords: Stage II/III colorectal cancer, Recurrence, Gene signature, Adjuvant chemotherapy, LASSO

*Correspondence: fccsunzq@zzu.edu.cn; fcchanxw@zzu.edu.cn

${ }^{1}$ Department of Interventional Radiology, The First Affiliated Hospital of Zhengzhou University, Zhengzhou 450052, Henan, China

${ }^{6}$ Department of Colorectal Surgery, The First Affiliated Hospital of Zhengzhou University, Zhengzhou 450052, Henan, China

Full list of author information is available at the end of the article

\section{Background}

According to the latest global cancer statistics, colorectal cancer (CRC) remains the third most common cancer and second leading cause of cancer-associated death worldwide [1]. The current stage of CRC is classified based on the tumor-node-metastasis (TNM) system issued by the American Joint Committee on Cancer (AJCC), which is routinely standard for the prognostic

(c) The Author(s) 2021. This article is licensed under a Creative Commons Attribution 4.0 International License, which permits use, sharing, adaptation, distribution and reproduction in any medium or format, as long as you give appropriate credit to the original author(s) and the source, provide a link to the Creative Commons licence, and indicate if changes were made. The images or other third party material in this article are included in the article's Creative Commons licence, unless indicated otherwise in a credit line to the material. If material is not included in the article's Creative Commons licence and your intended use is not permitted by statutory regulation or exceeds the permitted use, you will need to obtain permission directly from the copyright holder. To view a copy of this licence, visit http://creativecommons.org/licenses/by/4.0/. The Creative Commons Public Domain Dedication waiver (http://creativecommons.org/publicdomain/zero/1.0/) applies to the data made available in this article, unless otherwise stated in a credit line to the data. 
management and determination of adjuvant chemotherapy (ACT) of early-stage CRC [2]. After surgery, ACT is a conventional therapy for stage III and a subset of stage II CRC patients (eg, high grade, T4) [3, 4]. The goal of $\mathrm{ACT}$ is to eradicate residual cancer cells after surgical resection, thus reducing the recurrence rate or extending the time to recurrence. However, a consider number of patients with stage II/III CRC relapse or suffer from drug side effects, and also, current guidelines declare that the present definition of "high-risk" stage II CRC is inadequate [5-7]. Previous studies have demonstrated that approximately half of stage III CRC patients will relapse within 5 years after surgical resection, while the 5-years recurrence rate of stage II CRC patients is about $12-38 \%$ [8-10]. In clinical practice, the AJCC stage system alone is limited due to patients within the same stage have heterogeneous clinical outcomes [11]. Therefore, it is essential to redefine the risk stratification for recurrence in order to identify stage II/III CRC patients who may truly require or can omit $\mathrm{ACT}$.

Over the past few decades, intensive effort has been dedicated towards searching for new ways to evaluate the recurrence-risk of patients with early-stage CRC. We have previously demonstrated that the mutational status of TTN/OBSCN is an independent prognostic factor in CRC [12]. The mutation of BRAF, KRAS, and PIK3CA, loss of SMAD4, and amplification of HER2, are also reported to dramatically correlated with the recurrence of CRC [13-17]. Recently, Tie et al. have shown that circulating tumor DNA signature can serve as biomarkers of recurrence and benefit of ACT in stage III CRC [18]. In addition, the transcriptome-based consensus molecular subtype (CMS) and transcriptomic-based CRC intrinsic subtype (CRIS) systems have been reported to correlate with clinical outcomes in stage II/III CRC, and CMS4 or CRIS-C tumors have dismal recurrence and overall survival [19-21]. In parallel, patients with a low-frequency microsatellite instability (MSI-L) preferentially show a significantly increased risk of death and recurrence [22]. However, these classifiers only have a moderate prediction accuracy and limited clinical usefulness [23, 24].

Notably, an immunohistochemistry-based scoring pipeline has been established and validated (termed Immunoscore ${ }^{\circledR}$ ), which quantifies the densities of two adaptive immune cells, $\mathrm{CD} 3+$ and $\mathrm{CD} 8+\mathrm{T}$ cells, in the core and invasive margin of tumor [24]. Although Immunoscore ${ }^{\circledR}$ displays a stable predictive power of prognosis in early-stage CRC, its performance remains at a moderate accuracy of Harrell's C-statistics ranging from 0.56 to 0.68 in international researches [24]. This may be due to the fact that only two adaptive immune cells are considered, but other components in tumor may also be vital for evaluating recurrence-risk of early CRC. Our hypothesis is that comprehensive identification of key recurrence-associated genes and construction of predictive model will improve the accuracy of recurrence-risk assessment in stage II/III CRC. Traditional techniques such as immunohistochemistry or quantitative real-time polymerase chain reaction (qRT-PCR) are inconvenient to quantify a remarkable number of genes, but advances in bioinformatics have made it possible. Nowadays, we can easily obtain a large scale of genes for downstream analysis. With the help of machine learning, such as the least absolute shrinkage and selection operator (LASSO) algorithm, it is possible to identify the most important elements based on the expression profiles of global genes and fit a model with strong generalization performance [25].

In the present study, using four independent public cohorts with gene expression and recurrence-free survival (RFS) data, we performed a systematic and comprehensive biomarker discovery and validation work to develop a CRC recurrence-risk score (CRRS) system for predicting the RFS of patients with stage II/III CRC. Furthermore, we used 66 frozen tissue samples with qRT-PCR data for experimental verification to prove the stability and reliability of CRRS. Herein, we report a novel six-gene signature, which not only offers stable and excellent accuracy in identifying patients at high recurrence-risk, but also can be readily translated into the clinical practice due to the simplicity and inexpensiveness of PCR-based assays. Overall, we believe CRRS offers an attractive platform for evaluating recurrence-risk of patients with stage II/III CRC, and has important significance in optimizing decision-making in post-operative surveillance and therapeutic regimens.

\section{Methods}

\section{Public data collection and processing}

Four retrospective CRC cohorts were enrolled from Gene Expression Omnibus (GEO, http://www.ncbi.nlm. nih.gov/geo) database, including GSE143985, GSE17536, GSE29621, and GSE92921. These cohorts all belong to the Affymetrix ${ }^{\circledR}$ GPL570 platform ([HG-U133 Plus 2] Affymetrix $^{\circledR}$ Human Genome U133 Plus 2.0 Array). The raw data from Affymetrix ${ }^{\circledR}$ were processing using the robust multiarray averaging (RMA) algorithm implemented in the affy $R$ package. RMA was used to perform background adjustment, quantile normalization, and final summarization of oligonucleotides per transcript using the median polish algorithm. In four cohorts, we only retained CRC patients that met the following criteria: (1) Primary tumor tissues samples; (2) In the AJCC stage II/III; (3) Have both recurrent status and RFS information; (4) No preoperative chemotherapy or radiotherapy received. A total of 91 patients from GSE143985 
were used as the training set, and GSE17536 $(\mathrm{n}=111)$, GSE29621 $(\mathrm{n}=40)$, and GSE92921 $(\mathrm{n}=59)$ were used as the validation sets. The baseline data were summarized in Additional file 1: Table S1. The time from surgery to cancer recurrence was defined as RFS.

\section{Signature generation}

First, based on univariate Cox regression, genes with $P<0.05$ and hazard ratio (HR) consistently $>1$ or $<1$ in all enrolled cohorts were defined as stable recurrenceassociated genes. Next, using the expression of these stable recurrence-associated genes in training set, we developed a CRC recurrence-risk score (CRRS) system to predict the RFS of patients with stage II/III CRC via the LASSO Cox regression algorithm. By ten-fold cross validation, the optimal lambda was generated when the partial likelihood deviance reached the minimum value. Finally, based on the optimal lambda, genes with nonzero coefficients were selected to establish the prediction model. The CRRS for each patient was calculated with the LASSO model weighting coefficient as follows:

$$
C R R S=\sum_{i=1}^{n} \operatorname{Exp}_{i} \times \text { Coef }_{i}
$$

where $n$ is the number of key genes, $\operatorname{Exp}_{i}$ is the expression of gene $i$, and $\operatorname{Coef}_{i}$ is the LASSO coefficient of gene $i$.

\section{Human tissue specimens and qRT-PCR analysis}

From January 2015 to December 2016, we collected a total of 66 frozen surgically resected CRC tissues with AJCC stage II/III at The First Affiliated Hospital of Zhengzhou University. Follow up was concluded five years after surgery. Detailed baseline data of CRC patients were displayed in Additional file 1: Table S1. Total RNA was isolated from CRC tissues using RNAiso Plus reagent (Takara, Dalian, China) according to the manufacturer's instructions. RNA quality was evaluated using a NanoDrop One C (Waltham, MA, USA), and RNA integrity was assessed using agarose gel electrophoresis. An aliquot of $1 \mu \mathrm{g}$ of total RNA was reversetranscribed into complementary DNA (cDNA) according to the manufacturer's protocol using the miRNA reverse transcription Kit (TaKaRa BIO, Japan). All cDNA samples were prepared for qRT-PCR. This project was approved by the Ethics Committee Board of The First Affiliated Hospital of Zhengzhou University. In the qRTPCR analysis, the enrolled 6 genes in the model were detected. qRT-PCR was performed using SYBR Assay I Low ROX (Eurogentec, USA) and SYBR ${ }^{\circledR}$ Green PCR Master Mix (Yeason, Shanghai, China). The expression value of the target genes was normalized to GAPDH, and then $\log 2$ transformed for subsequent analysis. The primer sequences of the included 6 genes and GAPDH were shown in Additional file 1: Table S2.

\section{Statistical analysis}

All data processing, statistical analysis, and plotting were conducted in R 4.0.3 software. Continuous variables were compared between two groups through the Wilcoxon rank-sum test. Fisher's exact test was applied to compare categorical variables. The patients were divided into high and low-risk groups based on the median risk score. The Kaplan-Meier method and the log-rank test were utilized to estimate the different RFS between two groups. The receiver operating characteristic curve (ROC) used to predict binary categorical variables was implemented using the $\mathrm{R}$ package pROC. The time-dependent area under the ROC (AUC) for survival variable were conducted by the $\mathrm{R}$ package timeROC. The $\mathrm{R}$ package rms was applied to plot calibration curves. All statistical tests were two-sided. $P<0.05$ was regarded as statistically significant.

\section{Results}

\section{Baseline characteristics of patients}

As displayed in Additional file 1: Table S1, we enrolled a total of 367 patients with stage II/III CRC from five independent multicenter cohorts. In GSE143985, there were 55 stage II and 36 stage III patients with a median RFS of 5.8904 years; the 1-, 2-, and 3-year recurrence rate were $9.9 \%, 12.1 \%$, and $15.4 \%$, respectively. In GSE17536, there were 55 stage II and 56 stage III patients with a median RFS of 3.0625 years; the 1-, 2-, and 3-year recurrence rate were $6.3 \%, 15.3 \%$, and $23.4 \%$, respectively. In GSE29621, there were 22 stage II and 18 stage III patients with a median RFS of 3.9379 years; the 1-, 2-, and 3-year recurrence rate were $7.5 \%, 10.0 \%$, and $15.0 \%$, respectively. In GSE92921, there were 43 stage II and 16 stage III patients with a median RFS of 5.7260 years; the 1-, 2-, and 3-year recurrence rate were $6.8 \%, 8.5 \%$, and $10.2 \%$, respectively. In qRT-PCR validation cohort, there were 40 stage II and 26 stage III patients with a median RFS of 3.9671 years; the 1-, 2-, and 3-year recurrence rate were $15.2 \%, 16.7 \%$, and $21.2 \%$, respectively.

\section{Establishment and validation of CRRS with stage II/III CRC in public datasets}

The workflow was shown in Fig. 1. In total, univariate Cox results of four cohorts identified 13 genes were stably associated with RFS (all $P<0.05$ ) (Additional file 1: Table S3). Based on the expression of these genes in GSE143985, we fitted a LASSO Cox regression model and identified 6 genes that were strongly predictive of RFS, encompassing ELMSAN1, KRT33B, NDRG1, PPP1R13L, PPP2R1B, 


\section{Step I: Data arrangement}

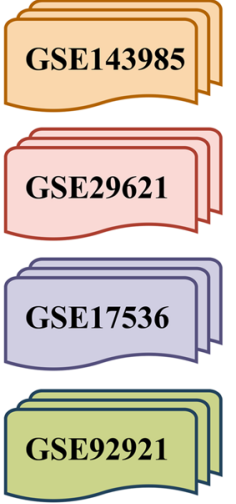

Inclusion Criteria:

(1) Primary tumor tissue samples;

(2) In the AJCC stage II/III;

(3) Have both recurrent status and RFS information;

(4) No preoperative chemotherapy or radiotherapy received.

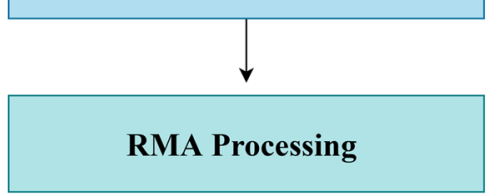

Step II: Establish the CRRS model

Detect stable recurrence-associated genes
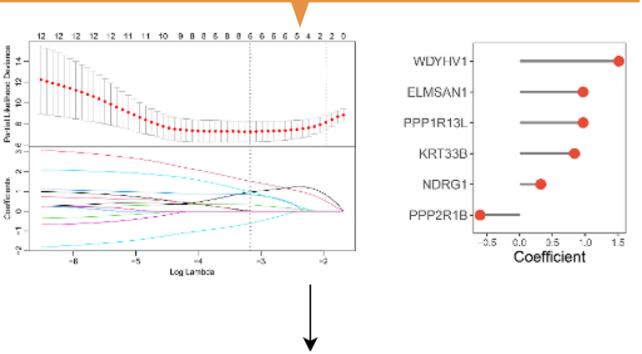

\section{Step III: Assess the predictive performance the CRRS model}
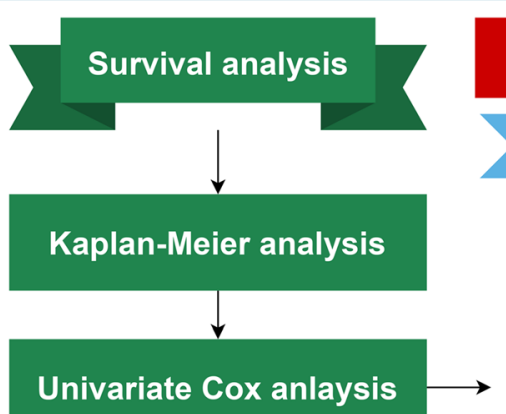

Univariate Cox anlaysis
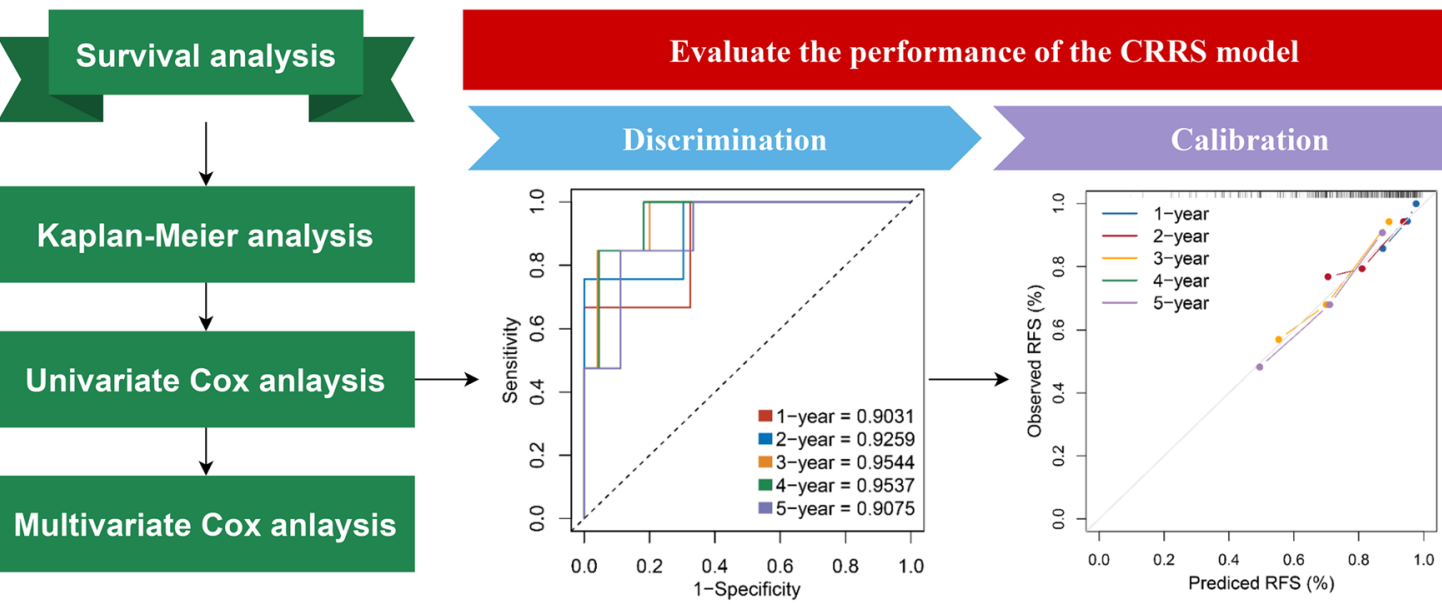

\section{Step IV: Validation of CRRS in a clinical cohort of 66 CRC patients by qRT-PCR assay}
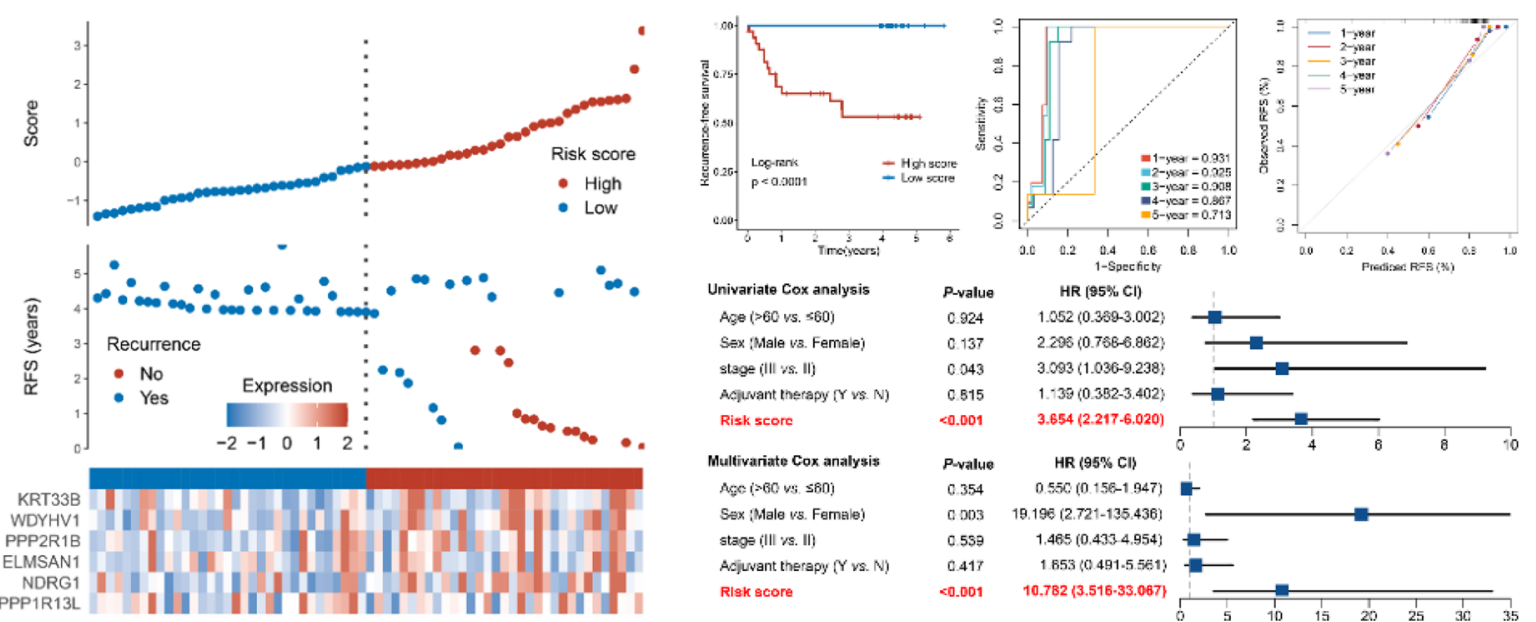

Univariate Cox analysis

A Sex : Male va. Femele; stage (III $v$. II) Aequant therapy (Y ve: N) Risk score

Multivariate $C_{0 x}$ analysis Age (D60 vis s60) Sex :Male va. Famale) stage ill vs. II! Acjuwant therapy $(Y$ vas. N) Rlsk score

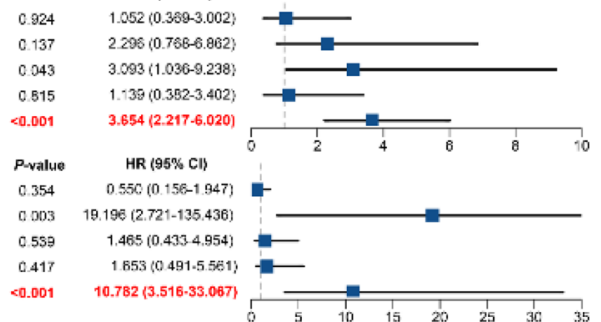

Fig. 1 The flowchart of this study 
and WDYHV1 (Fig. 2A). Next, the CRRS was calculated using a formula that including the 6 genes weighted by their regression coefficients in a penalized Cox model as follows: $C R R S=0.9698 \times \operatorname{Exp}(E L M S A N 1)+0.8381 \times \operatorname{Exp}$ $(K R T 33 B)+0.3238 \times \operatorname{Exp} \quad(N D R G 1)+0.9697 \times \operatorname{Exp}$ $(P P P 1 R 13 L)-0.6033 \times$ Exp $\quad(P P P 2 R 1 B)+1.5152 \times$ Exp (WDYHV1) (Fig. 2B). The CRRS of each patient calculated according to this formula. Expression heatmap of the 6 selected genes, distribution of CRRS, and recurrent status of each patient were illustrated in Fig. 2C. In four cohorts, all patients were segmented into high- and low-risk groups based on the median CRRS (Fig. 2C). Compared with the low-risk group, patients in the high-risk group displayed significantly unfavorable RFS in GSE143985 (HR: 4.296 [2.612-7.065], log-rank $P<0.0001$; Fig. 3A), GSE17536 (HR: 2.354 [1.662-3.334], log-rank $P<0.0001$; Fig. 3B), GSE29621 (HR: 3.934 [1.622-9.539], log-rank $P=0.0054$; Fig. 3C), and GSE92921 (HR: 7.080 [2.011-24.924], logrank $P=0.0110$; Fig. 3D) (Table 1). After adjusting the available clinical characteristics in four cohorts, multivariate Cox regression analysis revealed CRRS remained an independent risk factor for evaluating RFS of stage II/III CRC patients (all $P<0.01$ ) (Table 1 ).

\section{Predictive performance the CRRS model}

In this study, ROC and concordance index (C-index) as well as calibration curve were utilized to evaluate the discrimination and calibration of CRRS, respectively. The results showed that the AUCs for predicting RFS at $1-5$ years was $0.9399,0.9148,0.9274,0.9273$, and 0.9251 in GSE143985, 0.8070, 0.7113, 0.7698, 0.7697, and 0.7168 in GSE17536, 0.9031, 0.9259, 0.9544, 0.9537, and 0.9075 in GSE29621, and 0.9008, 0.9025, 0.9279, 0.9283, and 0.9267 in GSE92921, respectively (Fig. 4A). The C-index were 0.9018 [0.8396-0.9640], 0.7390 [0.6584-0.8196], 0.9279 [0.8256-1], and 0.9037 [0.8057-1] in four cohorts, respectively (Fig. 4B). These results suggested this model possessed high predative accuracy for predicting recurrence-risk at 1-5 years. Moreover, the CRRS showed excellent calibration, with the predicted probabilities of RFS at 1-5 years accurately, describing the true risk observed in all four cohorts (Fig. 4C). The CRRS also accurately separated the recurrence and recurrence-free CRC with tumor stage II/III after surgical resection. As illustrated in Fig. 4D, patients in the high-risk group displayed a significantly higher fraction of recurrence (high-risk vs. low-risk: $33 \%$ vs. $0 \%$ in GSE143985, $45 \%$ vs. $11 \%$ in GSE17536, 35\% vs. $0 \%$ in GSE29621, and 21\% vs. $\%$ in GSE92921; all $P<0.05$ ). It can be observed that CRRS perfectly separated recurrence and recurrence-free CRC in three cohorts. The ROC analysis further suggested the CRRS possessed high accuracy for identifying CRC patients with recurrence in all four cohorts (AUC: GSE143985 $=0.9360$, GSE17536 $=0.7911$, GSE29621 $=0.9524$, GSE92921 $=0.9277$ ) (Fig. 4E). Overall, in four public cohorts, the CRRS presented stable and excellent performance in evaluating RFS in patients with stage II/III CRC after surgical resection.

\section{Validation of CRRS in a clinical in-house cohort}

In order to verify the power of our six-gene CRRS model into a clinically translatable risk-stratification assay, we further performed qRT-PCR assays for these genes in a clinical cohort containing 66 CRC patients. Expression heatmap of the 6 selected genes, distribution of CRRS, and recurrent status of each patient were illustrated in Additional file 2: Fig. S1A. In line with our discovery insilico validation cohorts, patients with high score have the significantly dismal RFS (HR: 3.363 [2.093-5.404], $\log$-rank $P<0.0001$; Fig. 5A, B). Multivariate Cox regression analysis revealed that the CRRS remained the statistical significance (HR: 4.216 [2.283-7.784], $P<0.0001$; Fig. $5 \mathrm{~B}$ ), after adjusting for potential confounding factors (including age, sex, stage, and $\mathrm{ACT}$ ). The time-dependent ROC analysis showed the pinpoint accuracy of CRRS: the AUCs for predicting RFS at $1 \sim 5$ years was $0.931,0.925$, $0.908,0.867$, and 1.000, respectively (Fig. 5C). Likewise, the C-index reached 0.925 [0.862-0.988]. The calibration plot further displayed the predicted probabilities of RFS at 1-5 years accurately describing the true risk observed (Fig. 5D). The CRRS model perfectly distinguished recurrent CRC from non-recurrent CRC (high-risk vs. lowrisk: $42 \%$ vs. 0\%; Additional file 2: Fig. S1B), with a high precision $\mathrm{AUC}=0.919$ (Additional file 2: Fig. S1C). Of note, CRRS also showed a significantly higher accuracy than age, gender, stage, and chemotherapy (Additional file 2: Fig. S1D). Collectively, the results from a clinical in-house cohort supported that our discovery and in-silico validation cohort findings, which validated and confirmed that our CRRS model was quite robust, and can serve as an independent predictor of recurrence in stage II/III CRC.

(See figure on next page.)

Fig. 2 The development of the CRRS model based on the LASSO algorithm. A Ten-fold cross-validations to tune the parameter selection in the LASSO model. The two dotted vertical lines are drawn at the optimal values by minimum criteria (left) and 1 - SE (standard error) criteria (right). B LASSO coefficient profiles of the candidate genes for CRRS construction. C The distribution of risk score, recurrence status, and gene expression panel in four cohort 

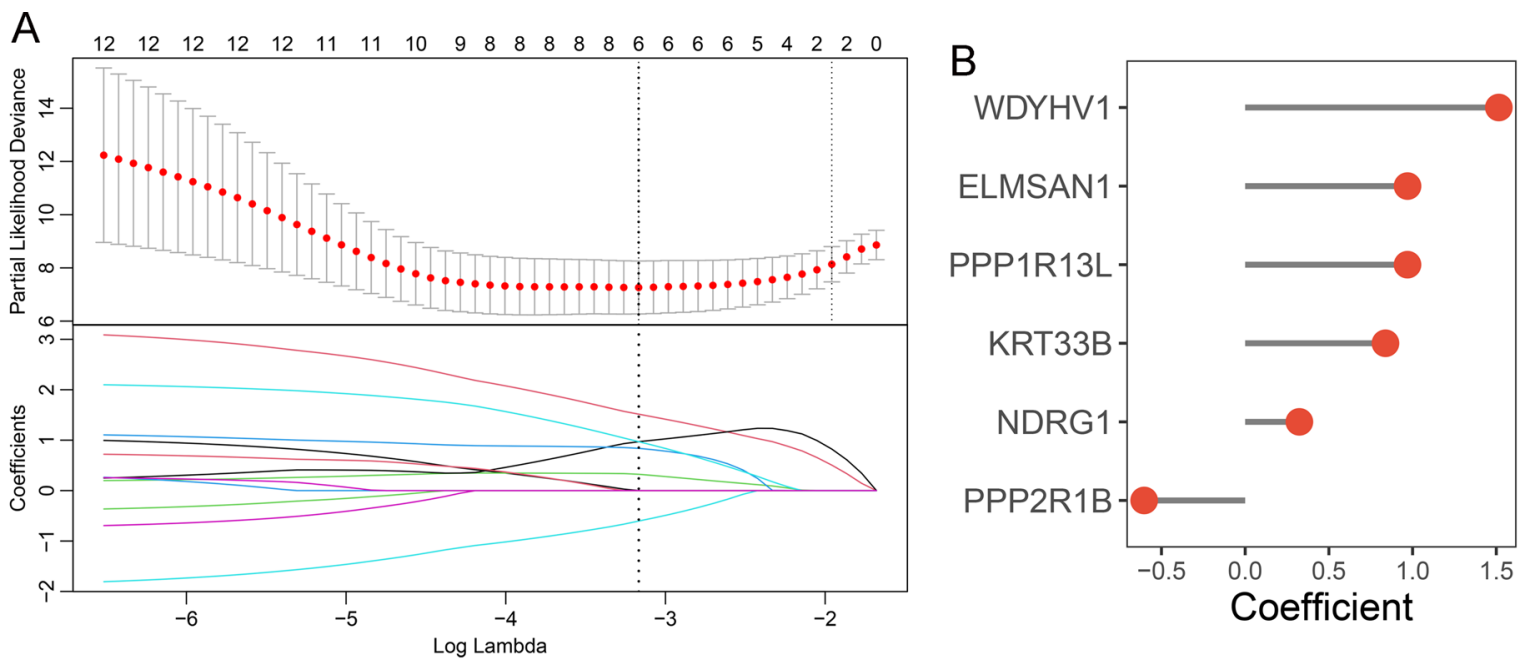

C

GSE143985

GSE17536
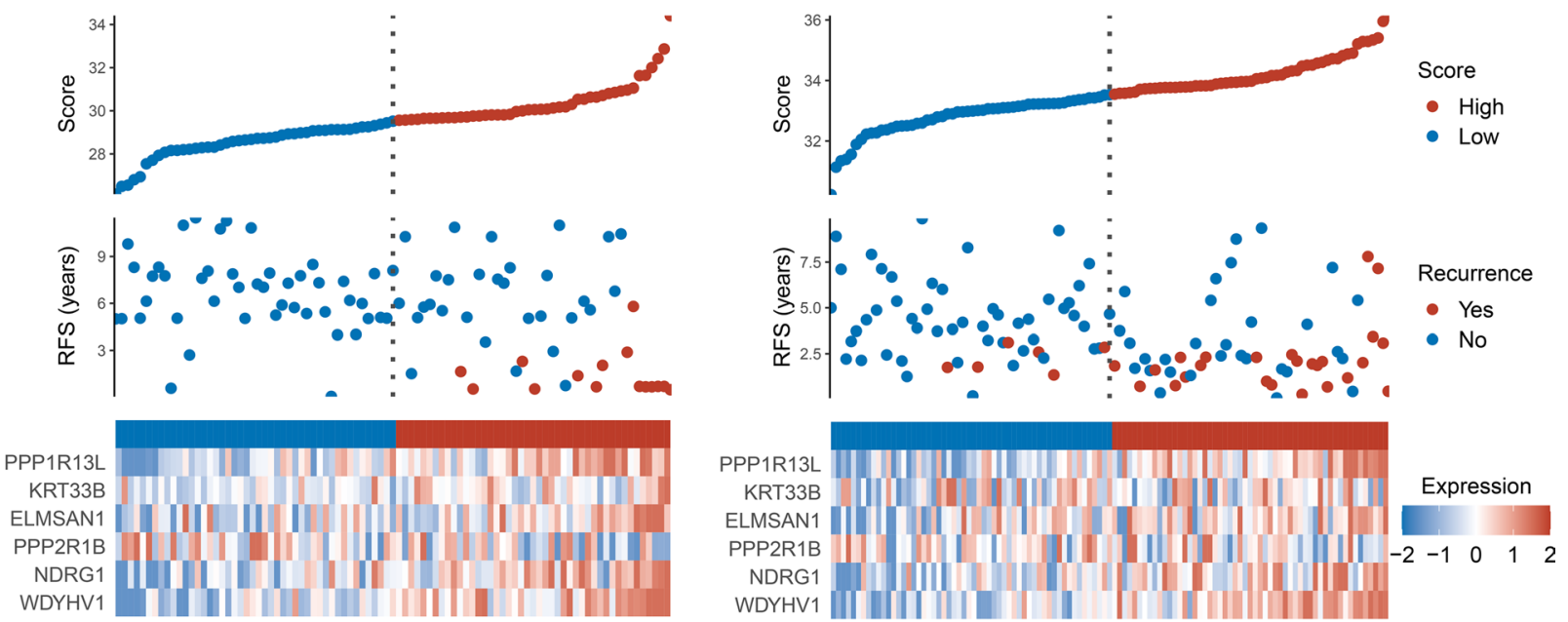

GSE29621
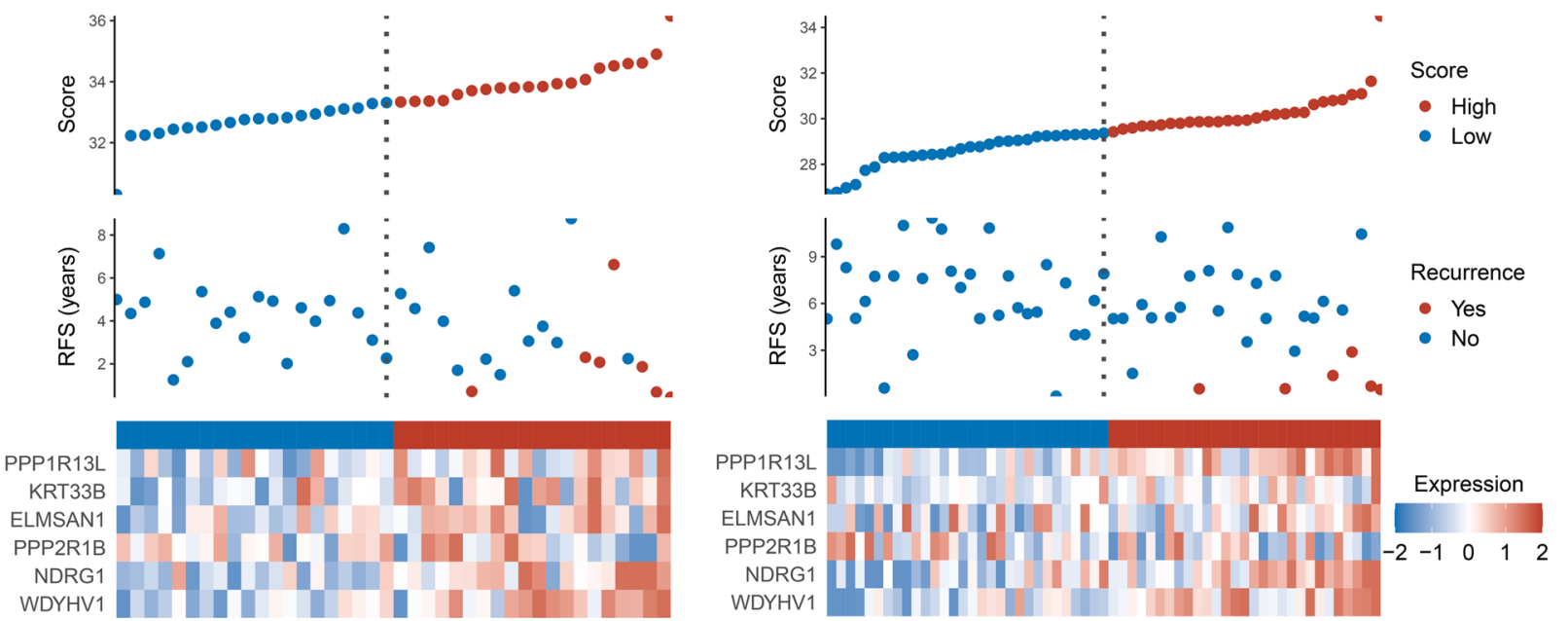

Fig. 2 (See legend on previous page.) 

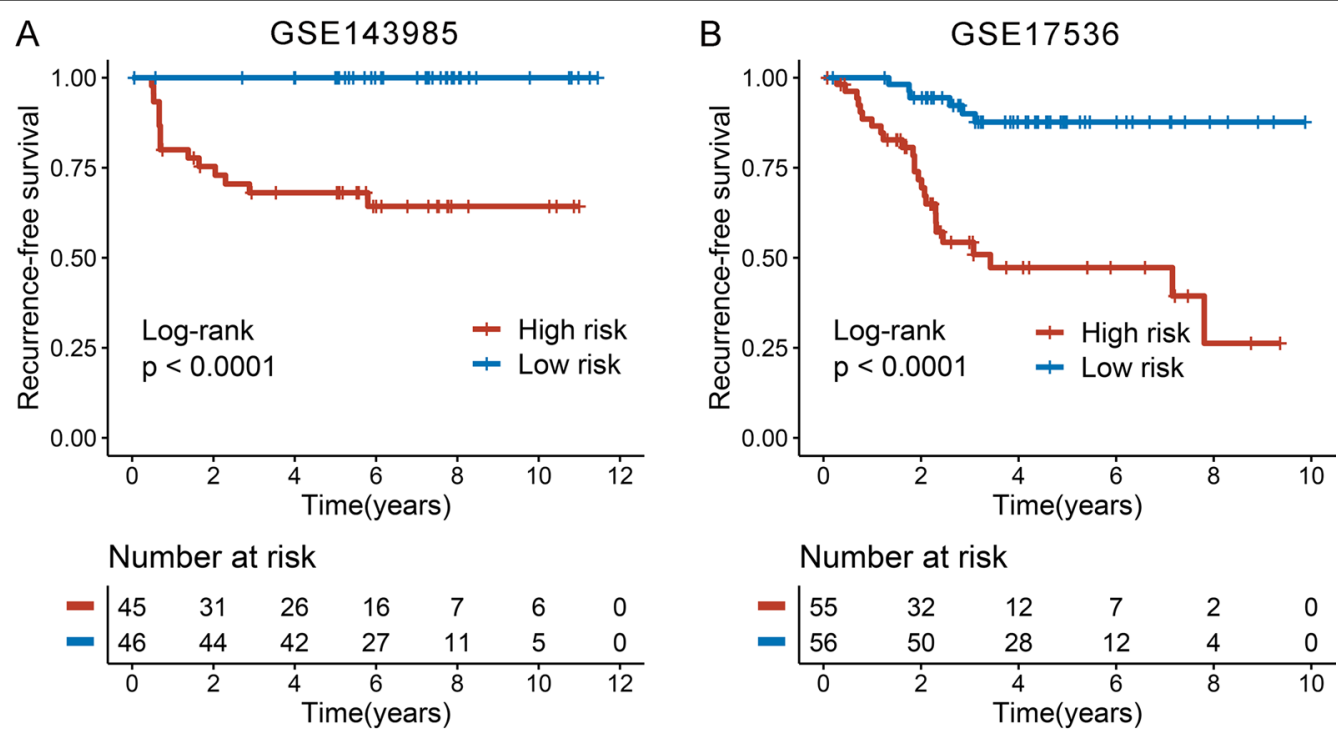

\section{Number at risk}
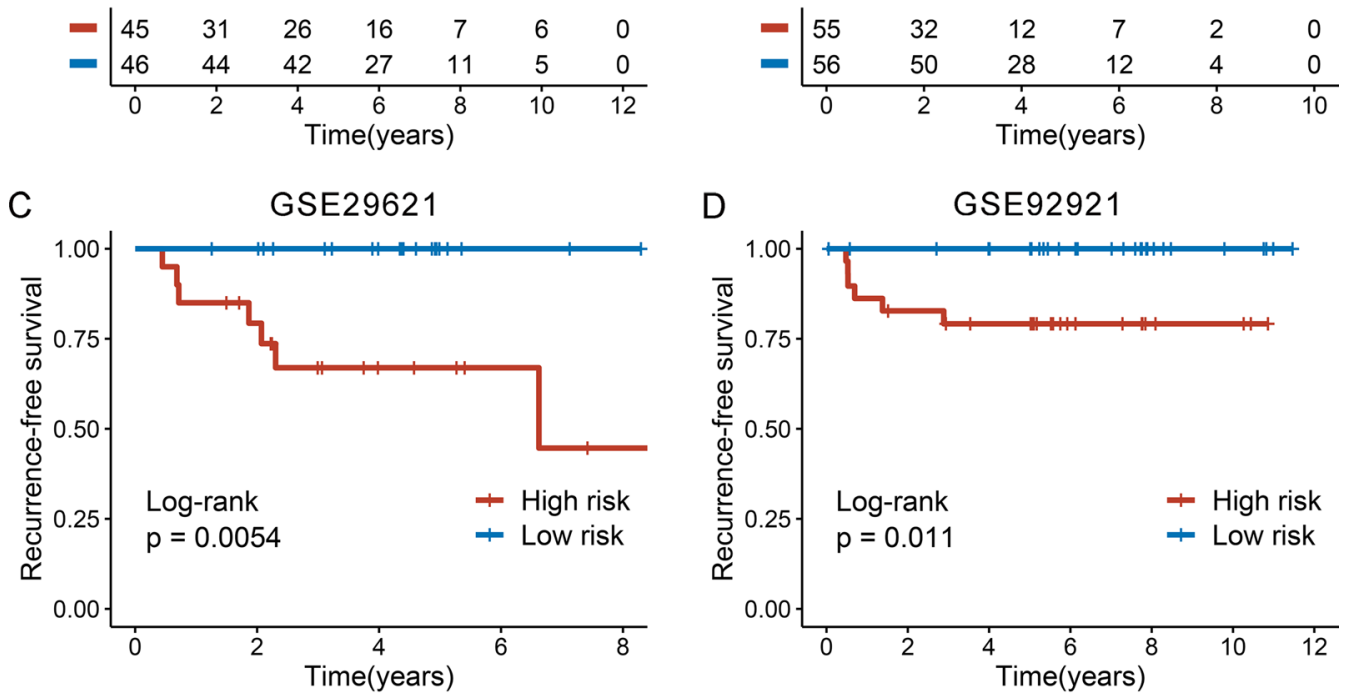

Number at risk

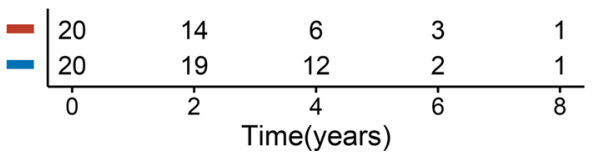

\section{Number at risk}

$=$\begin{tabular}{ccccccc}
29 & 23 & 20 & 9 & 4 & 3 & 0 \\
30 & 28 & 26 & 18 & 8 & 4 & 0 \\
\hline 0 & 2 & 4 & 6 & 8 & 10 & 12 \\
\multicolumn{7}{c}{ Time(years) }
\end{tabular}

Fig. 3 Kaplan-Meier survival analysis of CRRS in four cohorts. Kaplan-Meier curves of RFS according to the CRRS in GSE143985 (A), GSE17536 (B), GSE29621 (C), and GSE92921 (D)

\section{Discussion}

CRC is a highly heterogeneous tumor with complex biological processes and molecular mechanism, for which post-operative surveillance and therapeutic regimens are necessary to be tailored to generate an optimal outcome for each patient. Nevertheless, a considerable proportion of stage II/III CRC patients not only derive benefit from 5-Fluorouracil (5-FU)-based adjuvant chemotherapy but also display drug reactions $[5,6]$. A limitation of the current AJCC stage system is that patients in the same stage have distinct clinical outcomes, which leads to latent under- or over-treatment. Therefore, developing a novel classifier that can be routinely implemented into clinical practice is critical for identifying those earlystage patients who are at high recurrence-risk and who might thus benefit from adjuvant chemotherapy. We hypothesized that a signature with high performance could be developed according to the global immune milieu. With the development of artificial intelligence and bioinformatics, an advanced machine learning algorithm can identify several key indicators that are most meaningful to predict clinical outcomes from a large number of genes [25], which is actually in line with the biological scale-free network which was dominated by a few hub nodes [26]. Therefore, for the first time, we developed a novel signature (termed CRRS) to evaluate 
Table 1 Univariate and multivariate Cox regression analysis of the risk score

\begin{tabular}{|c|c|c|c|c|}
\hline \multirow[t]{2}{*}{ Characteristics } & \multicolumn{2}{|c|}{ Univariate Cox analysis } & \multicolumn{2}{|c|}{ Multivariate Cox analysis } \\
\hline & $\mathrm{HR}(95 \% \mathrm{Cl})$ & $P$-value & $\mathrm{HR}(95 \% \mathrm{Cl})$ & $P$-value \\
\hline \multicolumn{5}{|l|}{ GSE143985 } \\
\hline Stage (III vs II) & $4.058(1.272-12.943)$ & 0.018 & $6.876(1.276-37.041)$ & 0.025 \\
\hline$A C T(Y$ vs N) & $2.144(0.743-6.186)$ & 0.158 & $0.989(0.210-4.649)$ & 0.989 \\
\hline TP53 (Mut vs Wt) & $0.400(0.134-1.195)$ & 0.101 & $1.058(0.270-4.152)$ & 0.935 \\
\hline KRAS (Mut vs Wt) & $3.182(1.065-9.510)$ & 0.038 & $2.289(0.571-9.185)$ & 0.243 \\
\hline Risk score & $4.296(2.612-7.065)$ & $<0.001$ & $5.128(2.662-9.880)$ & $<0.001$ \\
\hline \multicolumn{5}{|l|}{ GSE17536 } \\
\hline Stage (III vs II) & $1.964(0.941-4.099)$ & 0.072 & $0.967(0.402-2.326)$ & 0.142 \\
\hline Age $(>60$ vs $\leq 60)$ & $0.482(0.238-0.978)$ & 0.043 & $0.728(0.308-1.721)$ & 0.009 \\
\hline Sex (Male vs Female) & $1.138(0.562-2.304)$ & 0.720 & $3.155(1.196-8.321)$ & 0.370 \\
\hline Risk score & $2.354(1.662-3.334)$ & $<0.001$ & $2.527(1.720-3.705)$ & $<0.001$ \\
\hline \multicolumn{5}{|l|}{ GSE29621 } \\
\hline Stage (III vs II) & $2.785(0.525-14.774)$ & 0.229 & $0.886(0.093-8.072)$ & 0.900 \\
\hline Age (> 60 vs $\leq 60)$ & $1.083(0.242-4.849)$ & 0.917 & $0.888(0.095-8.253)$ & 0.917 \\
\hline ACT (Y vs N) & $1.628(0.311-8.531)$ & 0.564 & $5.698(0.323-100.67)$ & 0.235 \\
\hline Risk score & $3.934(1.622-9.539)$ & 0.002 & $5.150(1.558-17.030)$ & 0.007 \\
\hline \multicolumn{5}{|l|}{ GSE92921 } \\
\hline Stage (III vs II) & $6.229(1.140-34.035)$ & 0.035 & $3.706(0.585-23.486)$ & 0.164 \\
\hline TP53 (Mut vs Wt) & $1.453(0.266-7.932)$ & 0.666 & $1.050(0.165-6.677)$ & 0.959 \\
\hline KRAS (Mut vs Wt) & $3.217(0.589-17.580)$ & 0.177 & $2.720(0.280-26.448)$ & 0.389 \\
\hline Risk score & $7.080(2.011-24.924)$ & 0.002 & $6.311(1.691-23.562)$ & 0.006 \\
\hline
\end{tabular}

recurrence-risk of patients with stage II/III CRC in multicenter cohorts. The reproducibility and powerful performance of CRRS in multiple independent cohorts and external qRT-PCR data not only prove that it is a robust and highly accurate model, but also is promising to be routinely implemented into clinical practice due to the advantages of high sensitivity and specificity, simplicity, and low cost of qRT-PCR.

In this study, we fitted a recurrence model consisting of 6 genes, including ELMSAN1, KRT33B, NDRG1, PPP1R13L, PPP2R1B, and WDYHV1 [27-31]. A majority of genes have been reported to be involved in the initiation and progression of tumor. For example, the downregulation of NDRG1 is associated with tumor metastasis via inducing epithelial-mesenchymal transition [28]; the expression of PPP $2 R 1 B$ was regulated by miRNA- 587 to antagonizes 5-FU-induced apoptosis in CRC [30]. Of note, the role of ELMSAN1 has not previously been reported in cancer, and thus requires further exploration. Based on the 6 enrolled genes, we developed the CRRS model, which performed stably in predicting recurrentrisk of patients with stage II/III CRC. The prognostic meta-analysis showed that RAIS was a risk indicator of recurrence and was proven to be an independent factor after adjusting multiple clinical clinicopathologic features. More importantly, in four cohorts, CRRS demonstrated a high discrimination and calibration in predicting the recurrence-risk at $1-5$ years. To prevent false positive results from sequencing data, we conducted another validation according to qRT-PCR results from 66 frozen CRC tissues with tumor stage II/III, confirming our prior findings and evaluating their practicality in different centers. As reported previously, patients with a high-risk score suggested dismal RFS, and thus might need to adjust therapy strategies or add additional adjuvant chemotherapy. For example, current guidelines recommend that a subset of stage II patients without "high-risk" traits do not require adjuvant chemotherapy [7], but when these patients show a high-risk score, using additional adjuvant chemotherapy might be essential.

Prior to this study, a few reports established molecular signatures for predicting prognostic risk of CRC [32-36]. In comparison with these studies, our work has several advantages and novelties: (1) The CRRS model was developed based on the recurrence rather than overall survival in patients with stage II/III CRC, which allowed it to accurately identify high-risk patients with earlystage CRC; (2) Fewer genes comprising the signature makes the CRRS easier to implement; (3) We performed comprehensive statistical approaches to evaluate the 

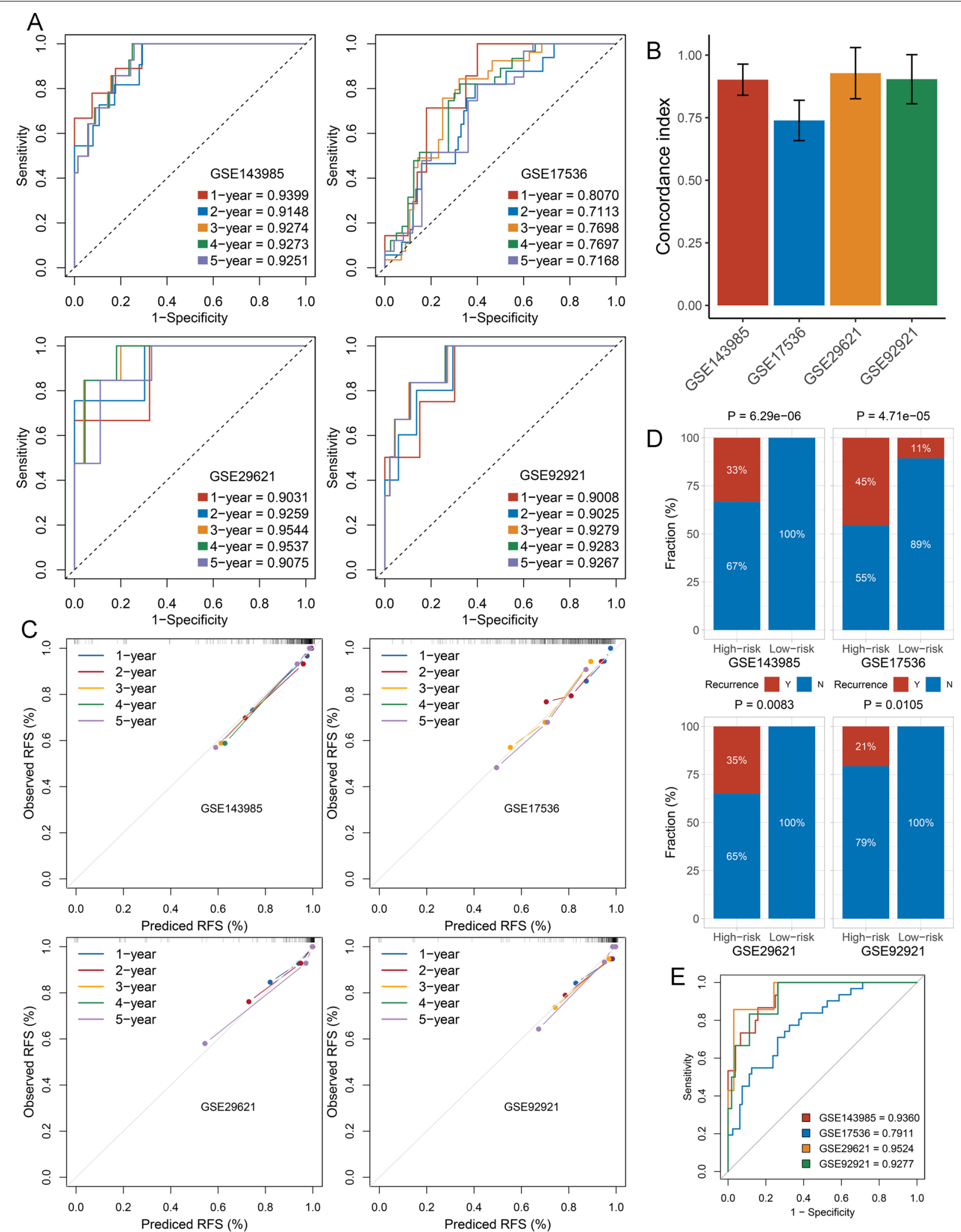

Fig. 4 Evaluation of the CRRS model in four cohorts. A Time-dependent ROC analysis for predicting RFS at $1 \sim 5$ years. B The Harrell's C-index of CRRS. C Calibration plots for comparing the actual probabilities and the predicted probabilities of RFS at 1 5 years. D Comparison of recurrence rate between the high-risk and low-risk groups. $\mathbf{E}$ ROC analysis of the CRRS model for predicting the recurrence event of patients 


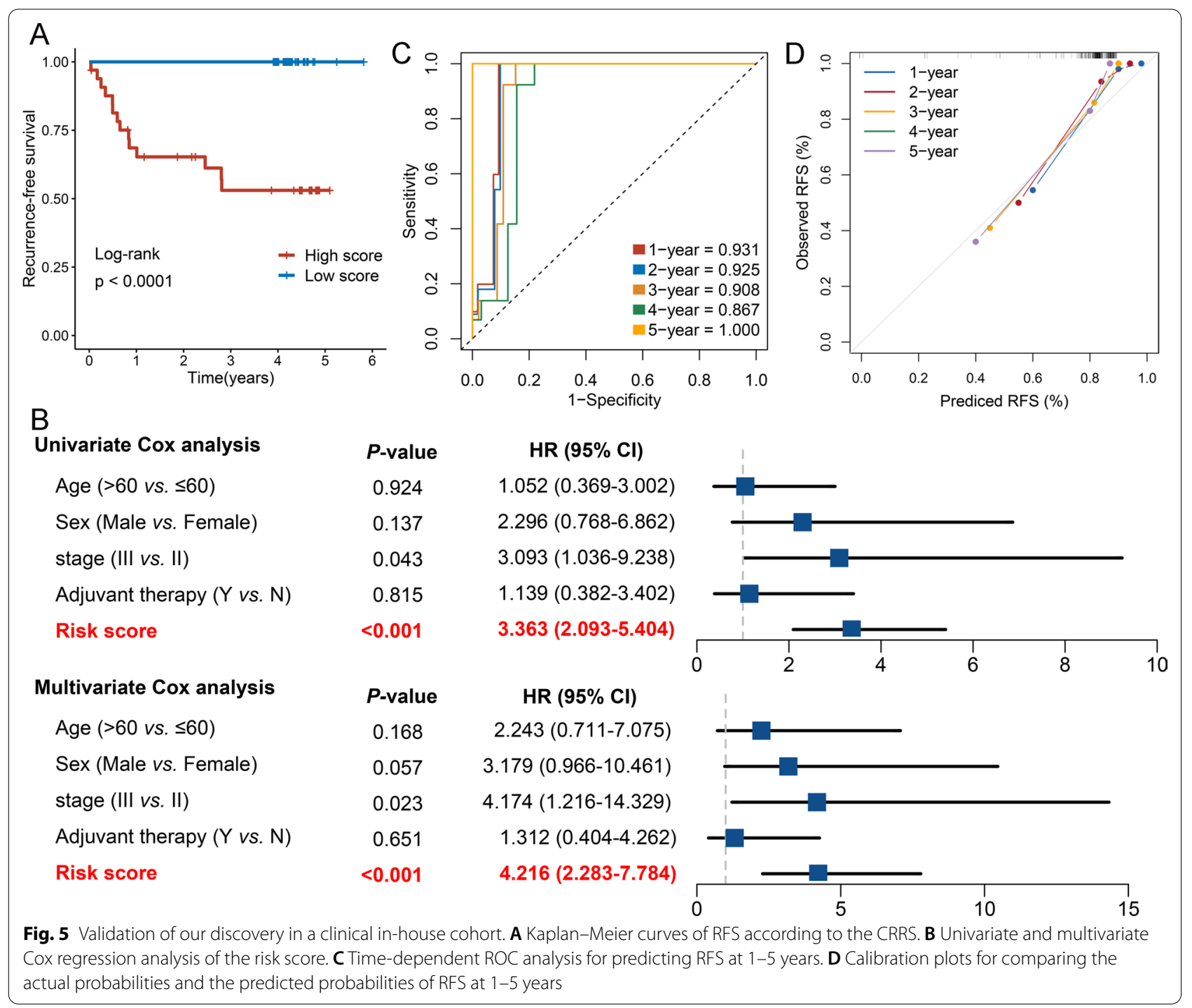

discrimination and calibration of the CRRS model, and our model remained stable and highly accurate performance at 1-5 years; (4) qRT-PCR was used to validate the performance of CRRS to ensure its robustness and clinical feasible. Despite the CRRS model is promising, some limitations should be acknowledged. First, all the samples from five centers were retrospective, and future validation of the CRRS model should be conducted in prospective fresh samples. Second, some clinical characteristics on public datasets were very inadequate, which thus had concealed the potential associations between CRRS and some clinical traits.

\section{Conclusions}

In summary, using a systematic and comprehensive biomarker discovery and validation approach, we established and validated a stable and powerful six-gene signature for evaluating the recurrence-risk of patients with stage II/III CRC. Our study demonstrated the CRRS model may be a promising tool to optimize decision-making in surveillance protocol and ACT for individual patients with stage II/III CRC.

\section{Abbreviations}

CRC: Colorectal cancer; TNM: Tumor-node-metastasis; AJCC: American Joint Committee on Cancer; ACT: Adjuvant chemotherapy; CMS: Consensus molecular subtype; CRIS: Intrinsic subtype; MSI-L: Low-frequency microsatellite instability; qRT-PCR: Quantitative real-time polymerase chain reaction; LASSO: Least absolute shrinkage and selection operator; RFS: Recurrence-free survival; CRRS: CRC recurrence-risk score; ROC: Receiver operating characteristic curve; AUC: Area under the ROC; C-index: Concordance index.

\section{Supplementary Information}

The online version contains supplementary material available at https://doi. org/10.1186/s12935-021-02070-z. 
Additional file 1: Table S1. Details of baseline information in GSE143985, GSE17536, GSE29621, GSE92921 and qRT-PCR data from 66 samples, respectively. Table S2. The forward and reverse primers for qRT-PCR. Table S3. Univariate Cox results of four cohorts revealed a total of 13 stable genes (red mark) were significantly associated with RFS.

Additional file 2: Fig. S1. Validation of CRRS in a clinical in-house cohort. A. The distribution of risk score, recurrence status, and gene expression panel in four cohort. B. Comparison of recurrence rate between the highrisk and low-risk groups. C. ROC analysis of the CRRS model for predicting the recurrence event of patients. D. C-index of CRRS, age, gender, stage, and chemotherapy for evaluating recurrence-free survival.

\section{Acknowledgements}

Not applicable.

\section{Authors' contributions}

ZQL and XWH designed this work. ZQL, QD, ZQS, JL, KHX, and DCJ integrated and analyzed the data. ZQL, TYL, LBW, CGG, and LL wrote this manuscript. ZQL, ZQS, JL, KHX, ZQS, and XWH edited and revised the manuscript. All authors approved this manuscript.

\section{Funding}

This study was supported by the National Natural Science Foundation of China (81972663); Henan Province Young and Middle-Aged Health Science and Technology Innovation Talent Project (YXKC2020037); and Henan Provincial Health Commission Joint Youth Project (SB201902014).

\section{Availability of data and materials}

Public data used in this work can be acquired from the TCGA Research Network portal (https://portal.gdc.cancer.gov/) and Gene Expression Omnibus (GEO, http://www.ncbi.nlm.nih.gov/geo/).

\section{Declarations}

\section{Ethics approval and consent to participate}

The human cancer tissues used in this study were approved by Ethnics Committee of The First Affiliated Hospital of Zhengzhou University in December 19, 2019, and the TRN is 2019-KW-423.

\section{Consent for publication}

Not applicable.

\section{Competing interests}

The authors declare that they have no competing interests.

\section{Author details}

'Department of Interventional Radiology, The First Affiliated Hospital of Zhengzhou University, Zhengzhou 450052, Henan, China. ${ }^{2}$ Interventional Institute of Zhengzhou University, Zhengzhou 450052, Henan, China. ${ }^{3}$ Interventional Treatment and Clinical Research Center of Henan Province, Zhengzhou 450052, Henan, China. ${ }^{4}$ Department of Cerebrovascular Disease, Zhengzhou University People's Hospital, Zhengzhou 450003, Henan, China. ${ }^{5}$ Department of Hepatobiliary and Pancreatic Surgery, The First Affiliated Hospital of Zhengzhou University, Zhengzhou 450052, Henan, China. ${ }^{6}$ Department of Colorectal Surgery, The First Affiliated Hospital of Zhengzhou University, Zhengzhou 450052, Henan, China. 'Department of Endovascular Surgery, The First Affiliated Hospital of Zhengzhou University, Zhengzhou 450052, Henan, China.

Received: 12 May 2021 Accepted: 29 June 2021

Published online: 07 July 2021

\section{References}

1. Sung H, Ferlay J, Siegel RL, Laversanne M, Soerjomataram I, Jemal A, et al. Global cancer statistics 2020: GLOBOCAN estimates of incidence and mortality worldwide for 36 cancers in 185 countries. CA Cancer J Clin. 2021;71(3):209-49.

2. Weiser MR. AJCC 8th edition: colorectal cancer. Ann Surg Oncol. 2018;25:1454-5.

3. Kannarkatt J, Joseph J, Kurniali PC, Al-Janadi A, Hrinczenko B. Adjuvant chemotherapy for stage II colon cancer: a clinical dilemma. J Oncol Pract. 2017;13:233-41.

4. Taieb J, Gallois C. Adjuvant Chemotherapy for Stage III Colon Cancer. Cancers (Basel). 2020;12:1.

5. Moertel CG, Fleming TR, Macdonald JS, Haller DG, Laurie JA, Goodman PJ, Ungerleider JS, Emerson WA, Tormey DC, Glick JH, Veeder MH. Levamisole and fluorouracil for adjuvant therapy of resected colon carcinoma. N Engl J Med. 1990:323:197-8.

6. Giacchetti S, Perpoint B, Zidani R, Le Bail N, Faggiuolo R, Focan C, et al. Phase III multicenter randomized trial of oxaliplatin added to chronomodulated fluorouracil-leucovorin as first-line treatment of metastatic colorectal cancer. J Clin Oncol. 2000;18:136-47.

7. Benson AB, Venook AP, Al-Hawary MM, Cederquist L, Chen YJ, Ciombor KK, et al. NCCN guidelines insights: colon cancer, Version 2.2018. J Natl Compr Canc Netw. 2018;16:359-69.

8. Carlsson U, Lasson A, Ekelund G. Recurrence rates after curative surgery for rectal carcinoma, with special reference to their accuracy. Dis Colon Rectum. 1987;30:431-4.

9. Dekker E, Tanis PJ, Vleugels JLA, Kasi PM, Wallace MB. Colorectal cancer. Lancet. 2019;394:1467-80.

10. Osterman E, Hammarstrom K, Imam I, Osterlund E, Sjoblom T, Glimelius B. Recurrence risk after radical colorectal cancer surgery-less than before, but how high is it? Cancers (Basel). 2020;12:1.

11. Benson AB 3rd, Hamilton SR. Path toward prognostication and prediction: an evolving matrix. J Clin Oncol. 2011;29:4599-601.

12. Liu Z, Wang L, Guo C, Liu L, Jiao D, Sun Z, et al. TTN/OBSCN “Double-Hit” predicts favourable prognosis, "immune-hot" subtype and potentially better immunotherapeutic efficacy in colorectal cancer. J Cell Mol Med. 2021;25(7):3239-51.

13. Margonis GA, Buettner S, Andreatos N, Kim Y, Wagner D, Sasaki K, et al. Association of BRAF mutations with survival and recurrence in surgically treated patients with metastatic colorectal liver cancer. JAMA Surg. 2018;153:e180996.

14. McCuaig S, Barras D, Mann EH, Friedrich M, Bullers SJ, Janney A, et al. The interleukin 22 pathway interacts with mutant KRAS to promote poor prognosis in colon cancer. Clin Cancer Res. 2020;26:4313-25.

15. Kato S, lida S, Higuchi T, Ishikawa T, Takagi Y, Yasuno M, et al. PIK3CA mutation is predictive of poor survival in patients with colorectal cancer. Int J Cancer. 2007;121:1771-8.

16. Wasserman I, Lee LH, Ogino S, Marco MR, Wu C, Chen X, et al. SMAD4 loss in colorectal cancer patients correlates with recurrence, loss of immune infiltrate, and chemoresistance. Clin Cancer Res. 2019;25:1948-56.

17. Richman SD, Southward K, Chambers P, Cross D, Barrett J, Hemmings $\mathrm{G}$, et al. HER2 overexpression and amplification as a potential therapeutic target in colorectal cancer: analysis of 3256 patients enrolled in the QUASAR, FOCUS and PICCOLO colorectal cancer trials. J Pathol. 2016;238:562-70.

18. Tie J, Cohen JD, Wang Y, Christie M, Simons K, Lee M, et al. Circulating tumor DNA analyses as markers of recurrence risk and benefit of adjuvant therapy for stage III colon cancer. JAMA Oncol. 2019;5(12):1710-7.

19. Guinney J, Dienstmann R, Wang X, de Reynies A, Schlicker A, Soneson C, et al. The consensus molecular subtypes of colorectal cancer. Nat Med. 2015;21:1350-6.

20. Isella C, Brundu F, Bellomo SE, Galimi F, Zanella E, Porporato R, et al. Selective analysis of cancer-cell intrinsic transcriptional traits defines novel clinically relevant subtypes of colorectal cancer. Nat Commun. 2017;8:15107.

21. Allen WL, Dunne PD, McDade S, Scanlon E, Loughrey M, Coleman H, et al. Transcriptional subtyping and CD8 immunohistochemistry identifies poor prognosis stage II/III colorectal cancer patients who benefit from adjuvant chemotherapy. JCO Precis Oncol 2018;2018. 
22. Petrelli F, Ghidini M, Cabiddu M, Pezzica E, Corti D, Turati L, et al. Microsatellite instability and survival in stage II colorectal cancer: a systematic review and meta-analysis. Anticancer Res. 2019;39:6431-41.

23. Dienstmann R, Villacampa G, Sveen A, Mason MJ, Niedzwiecki D, Nesbakken A, et al. Relative contribution of clinicopathological variables, genomic markers, transcriptomic subtyping and microenvironment features for outcome prediction in stage II/III colorectal cancer. Ann Oncol. 2019;30:1622-9.

24. Pages F, Mlecnik B, Marliot F, Bindea G, Ou FS, Bifulco C, et al. International validation of the consensus Immunoscore for the classification of colon cancer: a prognostic and accuracy study. Lancet. 2018;391:2128-39.

25. Tibshirani R. The lasso method for variable selection in the Cox model. Stat Med. 1997;16:385-95.

26. Zhang B, Horvath S. A general framework for weighted gene co-expression network analysis. Stat Appl Genet Mol Biol. 2005;4:17.

27. Liu F, Dong H, Mei Z, Huang T. Investigation of miRNA and mRNA co-expression network in ependymoma. Front Bioeng Biotechnol. 2020:8:177.

28. de Lima JM, Morand GB, Macedo CCS, Diesel L, Hier MP, Mlynarek A, et al. NDRG1 deficiency is associated with regional metastasis in ora cancer by inducing epithelial-mesenchymal transition. Carcinogenesis. 2020:41:769-77.

29. Laska MJ, Lowe SW, Zender L, Hearn S, Vogel U, Jensen UB, et al. Enforced expression of PPP1R13L increases tumorigenesis and invasion through p53-dependent and p53-independent mechanisms. Mol Carcinog. 2009:48:832-42.

30. Zhang Y, Talmon G, Wang J. MicroRNA-587 antagonizes 5-FU-induced apoptosis and confers drug resistance by regulating PPP2R1B expression in colorectal cancer. Cell Death Dis. 2015;6:e1845.
31. Asiedu MK, Thomas CF Jr, Dong J, Schulte SC, Khadka P, Sun Z, et al. Pathways impacted by genomic alterations in pulmonary carcinoid tumors. Clin Cancer Res. 2018:24:1691-704.

32. Smith JJ, Deane NG, Wu F, Merchant NB, Zhang B, Jiang A, et al. Experimentally derived metastasis gene expression profile predicts recurrence and death in patients with colon cancer. Gastroenterology. 2010;138:958-68.

33. Salazar R, Roepman P, Capella G, Moreno V, Simon I, Dreezen C, et al. Gene expression signature to improve prognosis prediction of stage II and III colorectal cancer. J Clin Oncol. 2011;29:17-24.

34. Jorissen RN, Gibbs P, Christie M, Prakash S, Lipton L, Desai J, et al. Metastasis-associated gene expression changes predict poor outcomes in patients with dukes stage $b$ and c colorectal cancer. Clin Cancer Res. 2009;15:7642-51.

35. Li J, Zhang J, Hu H, Cai Y, Ling J, Wu Z, et al. Gene expression signature to predict prognosis and adjuvant chemosensitivity of colorectal cancer patients. Cancer Manag Res. 2020;12:3301-10.

36. Kim SK, Kim SY, Kim JH, Roh SA, Cho DH, Kim YS, et al. A nineteen genebased risk score classifier predicts prognosis of colorectal cancer patients. Mol Oncol. 2014:8:1653-66.

\section{Publisher's Note}

Springer Nature remains neutral with regard to jurisdictional claims in published maps and institutional affiliations.
Ready to submit your research? Choose BMC and benefit from:

- fast, convenient online submission

- thorough peer review by experienced researchers in your field

- rapid publication on acceptance

- support for research data, including large and complex data types

- gold Open Access which fosters wider collaboration and increased citations

- maximum visibility for your research: over $100 \mathrm{M}$ website views per year

At BMC, research is always in progress.

Learn more biomedcentral.com/submissions 